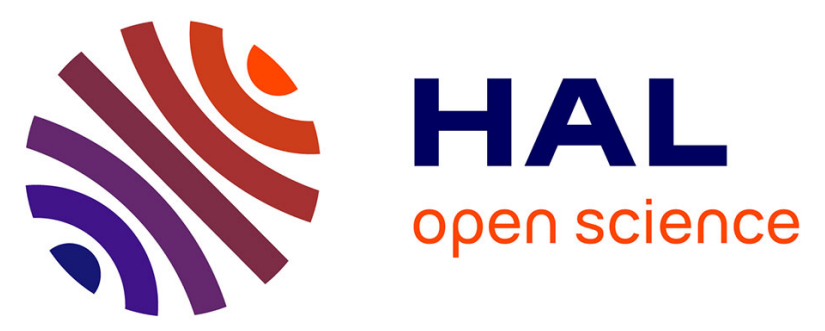

\title{
User-centered design of a multisensory power wheelchair simulator: towards training and rehabilitation applications
}

Guillaume Vailland, Fabien Grzeskowiak, Louise Devigne, Yoren Gaffary, Bastien Fraudet, Emilie Leblong, Florian Nouviale, François Pasteau, Ronan

Le Breton, Sylvain Guegan, et al.

\section{To cite this version:}

Guillaume Vailland, Fabien Grzeskowiak, Louise Devigne, Yoren Gaffary, Bastien Fraudet, et al.. User-centered design of a multisensory power wheelchair simulator: towards training and rehabilitation applications. ICORR 2019 - International Conference on Rehabilitation Robotics, Jun 2019, Toronto, Canada. pp.1-6. hal-02134530

\section{HAL Id: hal-02134530 \\ https://hal.inria.fr/hal-02134530}

Submitted on 20 May 2019

HAL is a multi-disciplinary open access archive for the deposit and dissemination of scientific research documents, whether they are published or not. The documents may come from teaching and research institutions in France or abroad, or from public or private research centers.
L'archive ouverte pluridisciplinaire HAL, est destinée au dépôt et à la diffusion de documents scientifiques de niveau recherche, publiés ou non, émanant des établissements d'enseignement et de recherche français ou étrangers, des laboratoires publics ou privés. 


\title{
User-centered design of a multisensory power wheelchair simulator: towards training and rehabilitation applications
}

\author{
Guillaume Vailland $^{1}$, Fabien Grzeskowiak ${ }^{1}$, Louise Devigne ${ }^{1}$, Yoren Gaffary ${ }^{1}$, \\ Bastien Fraudet ${ }^{2}$, Émilie Leblong ${ }^{2}$, Florian Nouviale ${ }^{1}$, François Pasteau ${ }^{1}$, Ronan Le Breton ${ }^{3}$, \\ Sylvain Guégan ${ }^{3}$, Valérie Gouranton ${ }^{1}$, Bruno Arnaldi ${ }^{1}$, Marie Babel ${ }^{1}$
}

\begin{abstract}
Autonomy and social inclusion can reveal themselves everyday challenges for people experiencing mobility impairments. These people can benefit from technical aids such as power wheelchairs to access mobility and overcome social exclusion. However, power wheelchair driving is a challenging task which requires good visual, cognitive and visuo-spatial abilities. Besides, a power wheelchair can cause material damage or represent a danger of injury for others or oneself if not operated safely. Therefore, training and repeated practice are mandatory to acquire safe driving skills to obtain power wheelchair prescription from therapists. However, conventional training programs may reveal themselves insufficient for some people with severe impairments. In this context, Virtual Reality offers the opportunity to design innovative learning and training programs while providing realistic wheelchair driving experience within a virtual environment. In line with this, we propose a user-centered design of a multisensory power wheelchair simulator. This simulator addresses classical virtual experience drawbacks such as cybersickness and sense of presence by combining 3D visual rendering, haptic feedback and motion cues. It relies on a modular and versatile workflow enabling not only easy interfacing with any virtual display, but also with any user interface such as wheelchair controllers or feedback devices. This paper presents the design of the first implementation as well as its first commissioning through pretests. The first setup achieves consistent and realistic behavior.
\end{abstract}

\section{INTRODUCTION}

People with disability may struggle to drive a power wheelchair because of altered perception skills. It has been shown that good visuo-spatial and cognitive abilities are necessary to achieve safe power wheelchair driving [1]. While training and repeated practice through occupational therapy sessions can be satisfactory for some people to master their wheelchair, this conventional training may be insufficient for others.

Some people may need training in specific situations such as driving in a urban environments or crowded places. However, such training set-ups raise safety issues and are difficult to carry out. Moreover, a lack of situation-specific training could lead to hazardous driving [2]. As a consequence, therapists can decide not to prescribe a power wheelchair for safety concerns (e.g. driving behavior is above a subjective risk level) [3].

In this context, wheelchair driving simulation can provide an additional training tool. Indeed, the beneficial effects

\footnotetext{
${ }^{1}$ Univ Rennes, INSA Rennes, CNRS, Inria, Irisa-UMR6074, France.

${ }^{2}$ Pôle Saint Hélier rehabilitation center, France.

${ }^{3}$ Univ Rennes, INSA Rennes, LGCGM EA 3913, France.
}

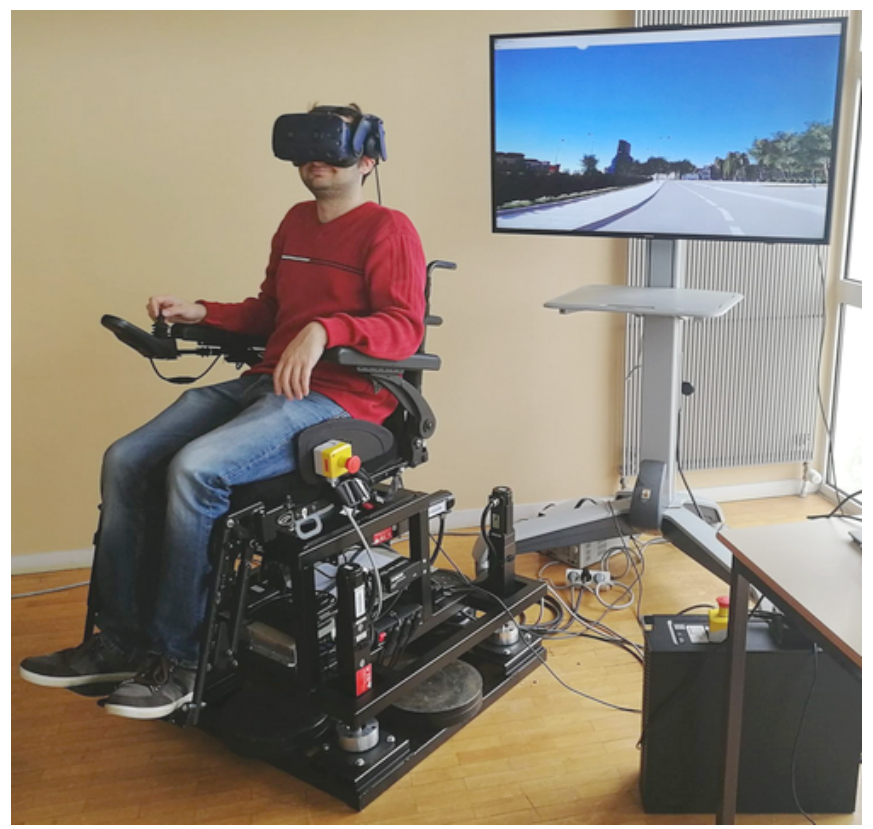

Fig. 1: The Power Wheelchair Simulator (PWS): motion platform and 3D scene displayed through HMD for an immersive experience in Rennes city

of training in simulation have been long recognized in applications such as aviation with flight simulators to train pilots [4]. Navigational skills acquired thanks to training in simulation have also been demonstrated to positively transfer into real situations for military training [5] or more recently for car driving training [6]. Moreover, simulation allows safe wheelchair navigation within controlled and repeatable scenarios [7].

Virtual Reality (VR) is one way of simulating ecological situations with daily-life scenarios which are hardly reproducible in a real world. While VR appears to be efficient and largely used in training programs [8] and rehabilitation [9], it is also been shown as being safe, easy to use, and contributing to high patient satisfaction [10]. In addition, VR allows therapists to achieve abilities assessment while reducing time, cost or safety constraints [11]. Besides, a recent study highlighted that VR can increase user excitement and motivation while performing a task in the virtual environment [9]. In the particular case of wheelchair driving, it has been shown that people with visuo-spatial and 
cognitive impairments may experience reduced motivation while driving a wheelchair [12]. VR could therefore reveal itself a more motivating tool for people with neurological disorders within wheelchair-related training programs.

A recent review on VR-based wheelchair simulators identified 29 wheelchair simulator projects of which 20 tackle power wheelchair simulation [13]. This review particularly highlighted the discrepancy among the proposed simulators. On the one hand, they differ in their designs and configuration setups. On the other hand, they differ in terms of assessment methodology and training programs, as most of the simulators were initially designed for driving training applications.

Two types of simulators can be identified in the literature. The first type of simulators incorporates a mechanical platform providing haptic and/or motion cues. For example, the Virtual Fauteuil simulator consists of a platform made of 4 actuators and a roller to reproduce manual wheelchair motion sensations [14]. Besides its focus on manual wheelchair simulation, the purpose of the simulator is to assess spatial planning and does not match clinical assessment requirement. The other type of simulators such that miWe [15] and ViEW [16] relies on driving scenario simulation displayed on a single screen and focus on the assessment of driving simulation impact on patients through learning and training programs. Despite the lack of haptic or motion cues, they have shown benefits on training.

Sense of Presence (SoP), defined as user's sense of "being there" in the simulation [17], has been shown to be a key-element for training performance [18]. Most VR-based simulators use 3D visual displays such as Head Mounted Devices (HMD) or immersive rooms to increase SoP. Some of the existing VR-based wheelchair driving simulators try to reach a higher SoP level by providing haptic feedback and/or motion cues in addition to the visual feedback. The major drawback of these simulators is that they are not compliant with clinical and user requirements and needs. Indeed, while the choice of the visual display should be adapted to user needs, they often rely on a specific visual display technology. Moreover, they do not provide easy transfer and adapted seating as they do not incorporate wheelchair standard components.

We propose a multi-sensory Power Wheelchair Simulator (PWS) designed in close collaboration with occupational therapists and wheelchair users. The proposed simulator includes a platform providing visual, auditory, and haptic feedback as well as motion cues. The proposed modular design allows to easily interface with any visual display (e.g. HMD, immersive rooms, single screen). It also allows easy integration of add-ons and configuration tuning to match individual user needs. The proposed PWS aims to provide realistic wheelchair driving experience for training and skill transfer to real-life situations.

This project is conducted by our multi-disciplinary team in Rennes gathering multiple research centers as INSA Rennes, Inria, IRISA and the Pôle Saint Hélier rehabilitation center. Thanks to this skill plurality, all our design choices have benefited from qualified experts' experience. In particular, computer and mechanical scientists worked closely with the clinical staff to better match therapists and wheelchair user needs and constraints.

This paper is organized as follows. Section III introduces the requirements that our proposed PWS must fulfill. Section III presents the design of our proposed PWS. Section IV details the implementation of our first PWS setup.

\section{Simulator REQUiREMENTS}

We propose a multisensory PWS design providing visual, auditory, haptic feedback and motion cue. This simulator aims to serve wheelchair driving training and clinical rehabilitation purposes. The simulator design is subject to constraints deduced from targeted user requirements but also from the VR technology itself.

\section{A. Targeted users requirements}

In order for the proposed PWS to be efficiently designed, end-user expertise must be considered. End-user needs were formulated thanks to therapists and patients from the Pôle Saint Hélier Physical Medicine and Rehabilitation center. This section therefore presents the constraints based on the recommendations of occupational therapists, physiotherapists, and neurologists but also and above all wheelchair users.

1) Seating and form factor: Considering the complexity of postural condition associated with some mobility impairments, a wide range of seating adjustments is needed. To avoid improperly fitted seating or positioning, the seating should be adjustable and dynamic, allowing for tilt and recline. Besides, a wide range of postural supports must be available on the seat based on the specific user needs, disability type, and postural condition. Moreover, the seat should include add-ons such as lateral trunk supports, hip guides, head rest, lateral thigh supports, armrests, and abduction block. A belt should also be used properly to secure participant positioning during simulation. Proper seating also requires footrests. The platform seat width and height must also be compliant to average wheelchair standards. Platform width must not be significantly larger than the seat width so it can be assimilated as a power wheelchair-like platform by its users.

2) Controller: The plurality of mobility impairments also implies a need for a wide range of control input adjustments, from proportional controllers (e.g. joysticks, chin controls) to discrete controllers (e.g. head array, sip-and-puff). Moreover, various input adaptations should be available for people with low upper limb strength or grasping difficulties (e.g. T-bar handle, U-bar handle, foam ball).

3) From power wheelchair to PWS kinematics: The objective is to provide a virtual driving experience similar to the one obtained with a power wheelchair. Therefore the simulator behavior has to accurately reproduce the dynamic behavior of a power wheelchair. Hence, we have identified the major kinematics parameters of power wheelchair motion 
(Fig. 2), e.g. the values of accelerations felt by a user (Table II).

These values have been experimentally obtained thanks to a set of driving scenarios representing classical indoor and outdoor use cases. In particular, these scenarios included curb and ramp climbing, navigation on rough surfaces, door passing...

The collected values correspond to a natural and safe navigation of a regular wheelchair user, and serve as reference. Dynamics occurring to accidental events, such as collisions and bumps, must not be fully reproduced by the motion feedback in order to alleviate the risks for users. These particular situations should be addressed through haptic and auditory feedback.

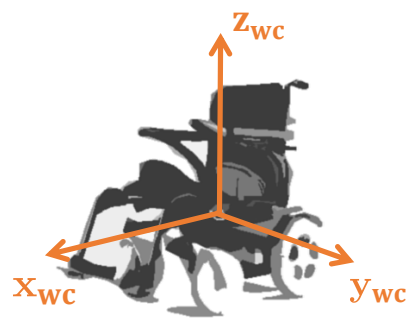

Fig. 2: Motion axes of a power wheelchair (wc).

\begin{tabular}{|c|c|c|c|}
\hline & $\begin{array}{c}\mathbf{x}_{\mathbf{w c}} \\
\text { direction }\end{array}$ & $\begin{array}{c}\mathbf{y}_{\mathbf{w c}} \\
\text { direction }\end{array}$ & $\begin{array}{c}\mathbf{z}_{\mathbf{w c}} \\
\text { direction }\end{array}$ \\
\hline $\begin{array}{c}\text { Translational } \\
\text { accelerations }\end{array}$ & $2 \mathrm{~m} . \mathrm{s}^{-2}$ & n.a. & $15 \mathrm{~m} . \mathrm{s}^{-2}$ \\
\hline $\begin{array}{c}\text { Rotational } \\
\text { accelerations }\end{array}$ & 2 rad.s $\mathrm{s}^{-2}$ & $10{\mathrm{rad} . \mathrm{s}^{-2}}^{-2}$ & $5 \mathrm{rad} . \mathrm{s}^{-2}$ \\
\hline
\end{tabular}

TABLE I: Maximum kinematics values of power wheelchair. The PWS should replicate these features.

4) Virtual experience: The visual display technology depends on each user specific needs. Therefore a simulator should not be dependent on a specific display device or technology and has to be compliant with various immersive visual displays such as HMDs and immersive rooms.

\section{B. VR technology requirements}

VR refers to a set of computer-based techniques aiming to simulate a 3D Virtual Environment (VE). As the proposed PWS relies on VR technology, VR-related constraints such as SoP and cybersickness must also be considered for our PWS design.

1) Sense of Presence ( $\mathrm{SoP}$ ): The SoP is a subjective phenomenon that has been defined in [17] as user's sense of "being there" in the simulation. It depends on various factors such as immersion, social interactions and 3D scene components (e.g. objects, avatars) behavior. The greater the SoP, the more realistically the user will behave. In that regard, SoP is a critical factor that our PWS must maximize.

2) Cybersickness: Immersion in a virtual environment can induce discomforts often characterized as cybersickness which symptoms are similar to motion sickness (e.g. nausea, headaches, dizziness) [19]. Cybersickness arises from perception conflicts between visual, vestibular and proprioceptive systems. Indeed, while moving around in the VE but getting no physical feedback or incorrectly configured feedback, users will get conflicting information. Cybersickness does not equally affect everyone. Moreover, targeted users with visual, visuo-spatial of cognitive impairments, are likely to be more sensitive to this symptoms. Those symptoms can significantly alter simulation comfort and can thus negatively impact simulator training efficiency. It is therefore critical to design a system minimizing cybersickness by reducing mismatches between visual, vestibular and proprioceptive information.

\section{Simulator DESIGN}

In previous work, we proposed a first wheelchair simulator design [20]. This simulator consisted in a standard power wheelchair fixed on the Immersia immersive room in Rennes, France. This first simulator matched the constraints related to clinical assessment presented in Section II-A as it directly used an off-the-shelf power wheelchair. In this framework, the user was seating on a motionless wheelchair and immersed in the VE through $3 \mathrm{D}$ rendering only. Although this first simulator generated a rather good SoP, most of the people who tested it felt cybersickness.

Based on this first simulator design, we here propose a new simulator design which aims to match VR-related constraints by maximizing SoP as well as addressing the cybersickness issue (Fig. 1).

\section{A. Towards a multisensory simulator}

While most of existing VR simulators use only 3D visual rendering techniques to immerse user, immersion is not restricted to visual sense only. Indeed, additional sensory information such as motion, visual, auditory [21] or even olfactory [22] cues can be simultaneously generated to improve user immersive experience. Then, we here propose an innovative multisensory simulator which incorporates a motion platform aiming to reproduce wheelchair motion cues and physical interactions with the virtual environment, as well as visual and auditory feedback modalities.

1) Motion platform: We design a motion platform generating motion cues in order to simulate accelerations as well as haptic feedback in order to reproduce interactions with the virtual environment such as bumps, vibrations and collisions.

Among the existing wheelchair simulators with motion platform, different types of simulators can be identified, depending on the number of degrees of freedom (DoF) that are used. In majority, simulators classically use either 3 DoF or 6 DoF (Stewart platform) [13]. If 6 DoF simulators achieve high frequency performances, 3 DoF simulators have been shown to be sufficient enough for training applications in terms of acceleration rendering.

In addition, some 6 DoF simulators add a turntable in order to extend rotation capabilities along vertical axis: this design achieves a high level of realism [23]. We then propose to add a turntable on a 3 DoF simulator, leading to an innovative 4 DoF simulator, able to reach kinematics requirements stated 

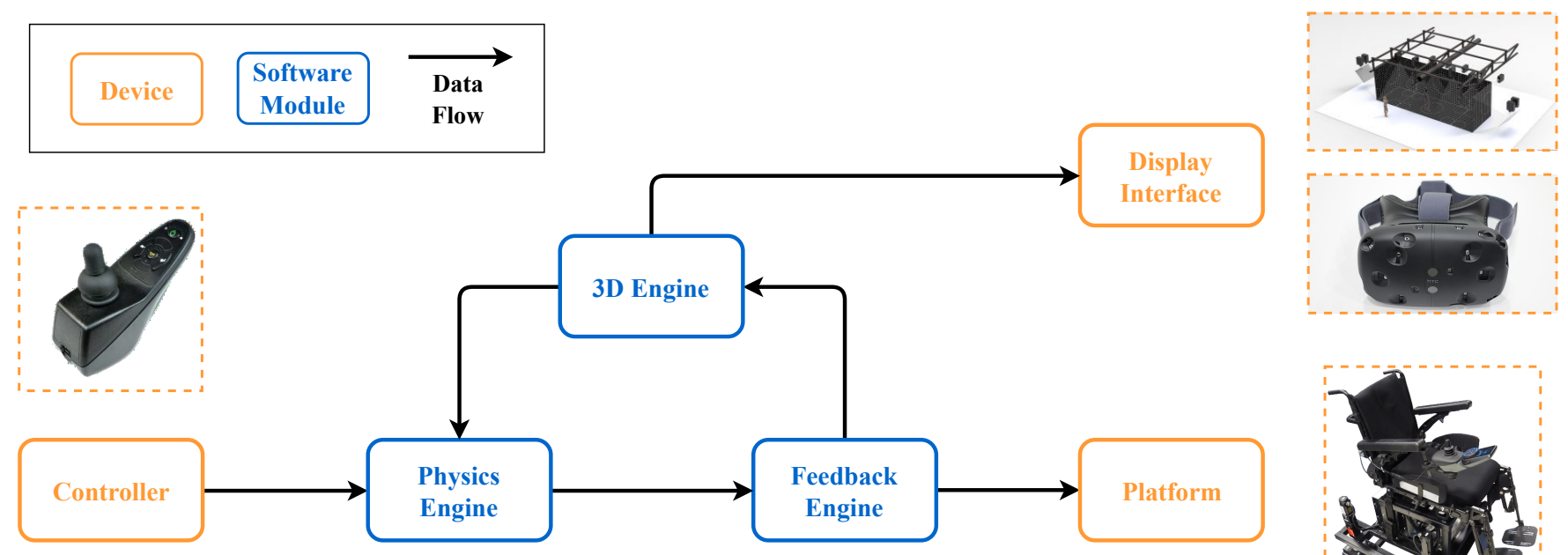

Fig. 3: PWS software diagram with examples of compliant devices

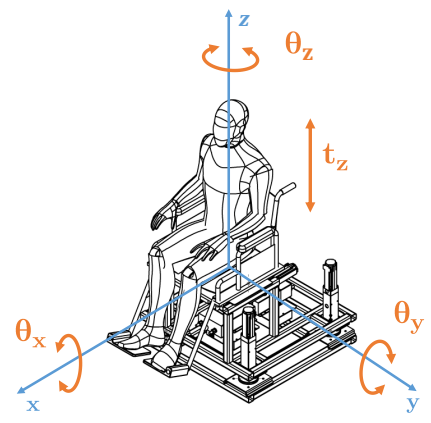

Fig. 4: Illustration of the 4 degrees of freedom of the simulator (in orange) related to the fixed reference frame (in blue)

in section $\Pi 1-\mathrm{A}$. The Fig. 4 illustrates the proposed solution, based on a parallel mechanism using 4 linear actuators (3 DoF) and a turntable on the top (4th DoF). This design is a trade-off between the desired range of motion and the compactness. Platform kinematic was designed according real power wheelchair behaviour [24].

An off-the-shelf SALSA M2 wheelchair seat from Sunrise Medical is then fixed on the turntable so that user environment does not differ from a commercial power wheelchair (see Fig. 5c): simulation experience is thus compliant with daily life experience.

The whole volume is similar to the one of a power wheelchair so that it increases the SoP for the use in immersive rooms. Also, the platform is lightweight in order to be compliant with immersive rooms. For example, the platform needs to be lighter than $100 \mathrm{~kg} / \mathrm{m}^{2}$ to match Immersia specifications. This choice will also ease the use and transport. The platform has been manufactured by CL Corporation (http://www.clcorporation.com/) from the specifications.

2) Visual feedback: Visual feedback provides wheelchair motion perception for each 6 DoF matching self-motion sensations suggested by the motion platform.
A virtual wheelchair is included in the $3 \mathrm{D}$ scenes and co-located with the motion platform. For instance, the 3D representation of the wheelchair seat in the VE matches the exact position of the motion platform seat.

Furthermore, the complexity of the virtual environment, in terms of feedback modalities, is adapted to user impairments and training programs. For example, as some user may not be able to process multiple visual stimuli, proposed 3D scenes would represent in these cases a low complex version of the scene.

3) Auditory feedback: Auditory feedback is provided to the user in order to increase immersive experience and SoP. This includes not only virtual sound rendered on speakers for ambient sounds, but also specific sound effects directly coming from the platform itself. In particular, the sound produced by the brakes of the wheelchair is an important auditory cue for the users: this sound is the first feedback perceived by users when a wheelchair starts to move.

\section{B. Simulator Workflow}

PWS design relies on a generic workflow (Fig. 3) composed of the following modules:

- The $3 D$ Engine manages the VE and its display.

- The Physics Engine updates the virtual wheelchair position, velocity and acceleration in the VE. These values are computed from input controller data, previous position, velocity and acceleration values sent by the $3 D$ Engine. It also incorporates physics laws (e.g. gravity).

- The Feedback Engine then computes the motion to be applied. The motion is split into a mechanical motion of the platform and to a visual feedback to the $3 D$ Engine.

These modules are independent, and dedicated to distinct specific tasks. This modular arrangement allows PWS to be a versatile and adaptable simulator. Each module can be concurrently and independently developed or replaced. The workflow presented here describes only required modules. 


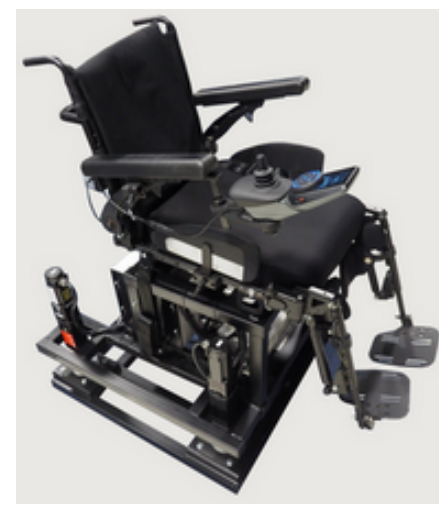

(a) PWS platform with wheelchair standard joystick

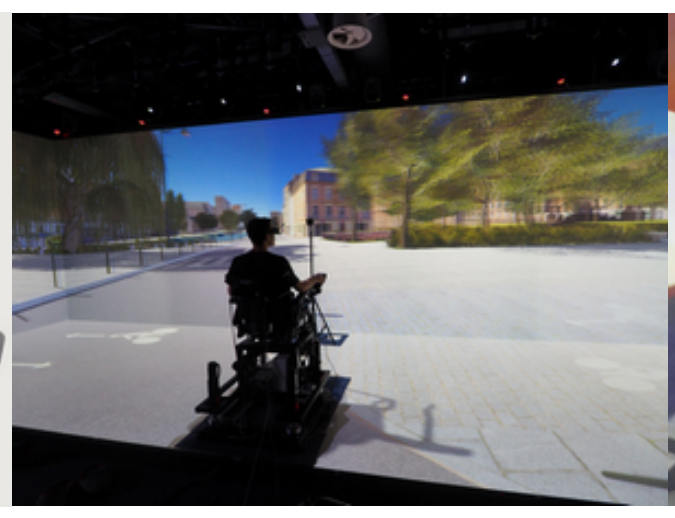

(b) PWS installed in Immersia with Rennes 3D scene - Courtesy of Rennes Metropole.

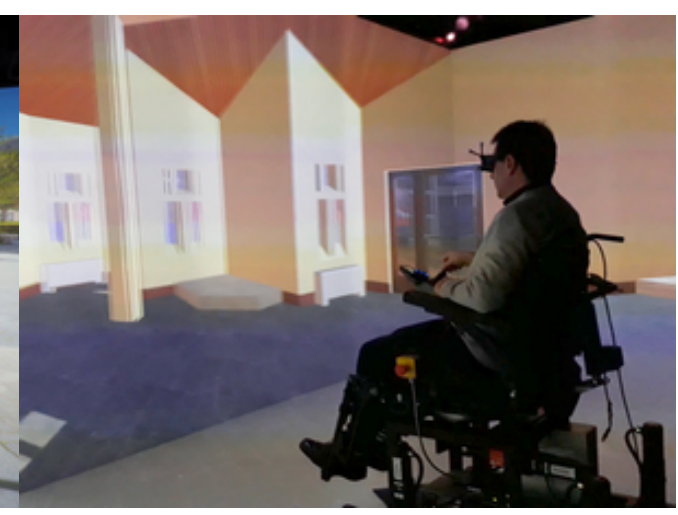

(c) PWS installed in Immersia with PSH experimental room 3D scene

Fig. 5: PWS first implementation

Several steps may be added without impacting the global process.

\section{SySTEM IMPLEMENTATION AND SETUP}

We developed, implemented and tested the following simulator setup.

1) Motion platform and motion cues: For our first experiments, only 2 degrees of freedom are used to provide motion cues. This feedback is based on "classical" algorithms as described in [25]. To manage with the lack of DoF, we included a "tilt coordination" strategy that implies a relationship with the 3D Engine as shown in the simulator workflow (Fig. 5c). An off-the-shelf wheelchair joystick controller is used as user interface.

2) Visual feedback and $3 D$ scenes: We used the widespread 3D engine Unity3D to visually render 3D scenes as it is compliant with several HMDs and Immersia (http: //www.irisa.fr/immersia/). In addition to their realism appearance, proposed 3D scenes are replication of real places known by end-users as real-like virtual environment are likely to increase user trust, comfort and SoP.

For now, our simulator includes two 3D scenes that are reproduction of real places. The first one is an outdoor environment: a urban area whose base model has been provided by Rennes Metropole. The second one is an indoor scene: a replication of Pôle Saint Hélier experimental room. Furthermore, because we simulate motion from a static spot with $4 \mathrm{DoF}$, it does not induce entirely real-like consequences. In particular, it causes undesired head movements leading to irregular gaze direction. Thus, we apply a visual compensation by moving the 3D scene accordingly.

3) System workflow: Finally, to implement a versatile workflow, we used the Robot Operating System (ROS) library [26]. Each module of the workflow is represented by a node in a ROS network that can send or receive data regardless of its execution environment and programming language. Thus, each node may be changed as long as input and output specifications are fulfilled.
Our first implementation has been successfully tested in several configurations. In particular, we tried PWS with two HMDs (HTC Vive and HTC Vive Pro), various screens and in Immersia (see Fig. 5b and 5a. As for the controller, we used 3 different commercial off-the-shelf joysticks. Regarding physical feedback, this platform achieves a consistent behaviour, which still needs tuning and more advanced studies.

\section{Discussion}

Our first simulator implementation is operational. In particular, the PWS has been successfully tested with different display devices (HMD, large immersive rooms, simple screens), 3D scenes (indoor/outdoor, low/high graphical resolution) and input devices (standard joystick, head array).

The motion platform as well as the simulator workflow have been designed to be versatile and adaptable not only to each user needs but also to rehabilitation center experimental environment and available hardware.

The proposed setup then meets previously stated usercentered constraints. The compliance of the system with wheelchair driving training and rehabilitation programs has now to be evaluated through clinical assessment with wheelchair users. In particular, the impact of the various feedback modalities provided by the simulator on SoP and cybersickness has to be assessed.

We are aware of the current PWS setup limitations and that some improvements still need to be made. On the one hand, regarding motion feedback, we plan to control all 4 DoF and to enhance the simulator kinematics in order to handle interactions with the environment such as bumps, vibrations and collisions. On the other hand, regarding the visual feedback and virtual environment, we want to populate 3D scenes with dynamic entities such as avatars, vehicles, etc. Furthermore, we want to add user embodiment in order to perform ecological situations as realistically as possible.

However, the simulator is ready to use, safe and includes a consistent motion feedback that can already be evaluated. Thus, we plan to conduct during the next months several experiments to assess the additional motion feedback impact 
on cybersickness and SoP (protocols similar to [20]). A study on the platform impact on cybersickness is currently being planned with about thirty able-bodied participants. A study of real power wheelchair dynamics is also planned in order to improve the virtual wheelchair dynamics estimations.

Besides studies and enhancements, we want to explore PWS additional use cases. For instance, based on our previous work on assistive robotics, we could consider adding virtual sensors and obstacles avoidance system in the simulator [20]. Thus, our simulator will be a convenient tool to prototype assistive systems.

\section{CONCLUSION}

In this paper we presented a user-centered design of a multisensory power wheelchair simulator. This simulator relies on VR technology to immerse the user in simulated wheelchair driving experience. The originality of the simulator relies on the combination of visual, auditory and motion cues provided by an innovative 4 degree of freedom mechanical structure. The proposed simulator design is versatile and adaptable to the diversity of impairments and needs. Indeed, it is directly compliant with any kind of 3D display devices that support Unity3D such as HMD, immersive room or screen display. In addition, wheelchair standard components fixed on the mechanical structure allow the platform to be compliant with clinical requirements. Indeed, the proposed simulator is directly compliant with wheelchair user seating and control needs. Clinical assessment of the simulator with power wheelchair users will then allow evaluation of driving experience in simulation but also its comparison between virtual and real conditions. In addition, the impact of the multimodal feedback provided by the simulator on user experience will be assessed in next studies.

\section{ACKNOWLEDGEMENT}

The authors would like to thank Ronan Gaugne from IRISA Immersia, Solenne Fortun from the IRISA Rainbow team and Éric Bazin from IETR/INSA.

This work is carried out as part of the INTERREG VA FMA ADAPT project funded by the European Regional Development Fund (ERDF), and is partially funded by the Crowdbot Project, a European Union Horizon 2020 Project.

\section{REFERENCES}

[1] S. Massengale, D. Folden, P. McConnell, L. Stratton, and V. Whitehead, "Effect of visual perception, visual function, cognition, and personality on power wheelchair use in adults," Assistive Technology, vol. 17, no. 2, pp. 108-121, 2005.

[2] R. C. Simpson, "Smart wheelchairs: A literature review," The Journal of Rehabilitation Research and Development, vol. 42, no. 4, pp. 423438, 2005.

[3] W. B. Mortenson, L. H. Clarke, and K. Best, "Prescribers ' Experiences With Powered Mobility Prescription Among Older Adults," American Journal of Occupational Therapy, vol. 67, no. 1, pp. 100-107, 2013.

[4] R. T. Hays, J. W. Jacobs, C. Prince, and E. Salas, "Flight simulator training effectiveness: A meta-analysis," Military psychology, vol. 4, no. 2, pp. 63-74, 1992.

[5] R. B. Loftin, M. W. Scerbo, F. D. McKenzie, and J. Catanzao, "Training in peacekeeping operations using virtual environments," IEEE Computer Graphics and Applications, vol. 24, no. 4, pp. 18$21,2004$.
[6] P. Hirsch and F. Bellavance, "Pilot project to validate the transfer of training of driving skills learned on a high fidelity driving simulator to on-road driving," Interuniversity Research Centre on Enterprise Networks, Logistics and Transportation, 2016.

[7] P. Abellard, I. Randria, A. Abellard, M. M. B. Khelifa, and P. Ramanantsizehena, "Electric wheelchair navigation simulators: why, when, how?," in Mechatronic Systems Applications, IntechOpen, 2010.

[8] D. Inman, K. Loge, and J. Leavens, "VR Education and Rehabilitation," Communications of the ACM, vol. 40, no. 8, pp. 53-59, 1997.

[9] M. C. Howard, "A meta-analysis and systematic literature review of virtual reality rehabilitation programs," Computers in Human Behavior, vol. 70, pp. 317-327, 2017.

[10] J. Dascal, M. Reid, W. W. Ishak, B. Spiegel, J. Recacho, and B. Rosen, "Virtual Reality and Medical Inpatients: A Systematic Review of Randomized, Controlled Trials," Innovations in clinical neuroscience, vol. 14 , no. 1-2, pp. 14-21, 2017.

[11] T. Ogourtsova, P. Archambault, S. Sangani, and A. Lamontagne, "Ecological Virtual Reality Evaluation of Neglect Symptoms (EVENS): Effects of Virtual Scene Complexity in the Assessment of Poststroke Unilateral Spatial Neglect," Neurorehabilitation and Neural Repair, vol. 32, no. 1, pp. 46-61, 2018.

[12] J. Cawood, S. Visagie, and G. Mji, "Impact of post-stroke impairments on activities and participation as experienced by stroke survivors in a Western Cape setting," South African Journal of Occupational Therapy, vol. 13, no. 3, pp. 153-160, 2016.

[13] S. Arlati, V. Colombo, G. Ferrigno, R. Sacchetti, and M. Sacco, "Virtual reality-based wheelchair simulators: A scoping review," Assistive Technology, pp. 1-12, 2019.

[14] F. Pouvrasseau, É. Monacelli, S. Charles, A. Schmid, F. Goncalves, P.-A. Leyrat, F. Coulmier, B. Malafosse, V.-v. France, F. Goncalves, P.-a. Leyrat, and B. Malafosse, "Discussion about functionalities of the Virtual Fauteuil simulator for wheelchair training environment," in International Conference on Virtual Rehabilitation (ICVR), pp. 17, 2017.

[15] P. S. Archambault, É. Blackburn, D. Reid, F. Routhier, and W. C. Miller, "Development and user validation of driving tasks for a power wheelchair simulator," Disability and Rehabilitation, vol. 39, no. 15 , pp. 1549-1556, 2016.

[16] Y. Morere, G. Bourhis, K. Cosnuau, G. Guilmois, E. Rumilly, and E. Blangy, "ViEW, a wheelchair simulator for driving analysis," Assistive Technology, pp. 1-11, 2018.

[17] M. Slater and S. Wilbur, "A framework for immersive virtual environments (FIVE): Speculations on the role of presence in virtual environments," Presence: Teleoperators \& Virtual Environments, vol. 6, no. 6, pp. 603-616, 1997.

[18] J. A. Stevens and J. P. Kincaid, "The relationship between presence and performance in virtual simulation training," Open Journal of Modelling and Simulation, vol. 3, no. 02, p. 41, 2015.

[19] L. Rebenitsch and C. Owen, "Review on cybersickness in applications and visual displays," Virtual Reality, vol. 20, no. 2, pp. 101-125, 2016.

[20] L. Devigne, M. Babel, F. Nouviale, V. K. Narayanan, F. Pasteau, and P. Gallien, "Design of an immersive simulator for assisted power wheelchair driving," IEEE International Conference on Rehabilitation Robotics, pp. 995-1000, 2017.

[21] W. R. Sherman and A. B. Craig, Understanding virtual reality: Interface, application, and design. Morgan Kaufmann, 2018.

[22] C. Porcherot, S. Delplanque, N. Gaudreau, M. Ischer, A. De Marles, and I. Cayeux, "Immersive techniques and virtual reality," in Methods in Consumer Research, Volume 2, pp. 69-83, Elsevier, 2018.

[23] T. Ito, M. Shino, T. Inoue, and M. Kamata, "Development of a powered wheelchair driving simulator for research and development use," Journal of Mechanical Systems for Transportation and Logistics, vol. 2, pp. 90-101, 012009.

[24] A. Baudry, S. Guegan, and M. Babel, "Taking caster wheel behavior into account in the kinematics of powered wheelchairs," Modelling, Measurement and Control C, vol. 79, pp. 168-172, 122018.

[25] F. Colombet, M. Dagdelen, G. Reymond, C. Pere, F. Merienne, and A. Kemeny, "Motion cueing: What is the impact on the driver's behavior ?," in Proceedings of the Driving Simulation Conference, pp. 171-182, 2008.

[26] M. Quigley, K. Conley, B. Gerkey, J. Faust, T. Foote, J. Leibs, R. Wheeler, and A. Y. Ng, "ROS: an open-source Robot Operating System," in ICRA workshop on open source software, no. 3, p. 5, 2009. 